\title{
Análisis del desarrollo de valores a través de la educación física y del deporte: un estudio de protocolo \\ Values development analysis through physical education and sports: a protocol study \\ Eulisis Smith-Palacio, Jorge Acebes-Sánchez \\ Universidad Francisco de Vitoria (España)
}

Resumen. Son muchas las teorías planteadas sobre la educación en valores y la transmisión de estos a través del deporte. No obstante, estas teorías han sido cuestionadas por la falta de evidencia en la metodología y en la identificación de variables que influyen en el desarrollo de los valores. Este estudio tiene como objetivo plantear el protocolo de intervención educativa del Programa Delfos para la educación en valores a través de actividades físico-deportivas en jóvenes escolares, y la evaluación del autocontrol a través del cuestionario de autocontrol infantil y adolescente (CACIA). Los resultados ayudarán a evaluar, si el programa de intervención Delfos es apropiado para el desarrollo de valores en el alumnado allá donde se aplique la investigación. Esta investigación pretende dotar al docente de herramientas para el desarrollo real de su alumnado en materia de valores a través de actividades físico-deportivas. Así mismo, el presente trabajo busca crear una metodología que permita transferir los valores educativos que se obtienen en las clases de educación física a la familia y la sociedad.

Palabras clave: Programa Delfos, educación física, agresividad, comportamientos, valores.

\begin{abstract}
There are many theories raised about education in values and the transmission of these through sport. However, these theories have been questioned by the lack of evidence in the methodology and in the identification of variables that influence the development of values. This study aims to propose the educational intervention protocol of the Delphi Program for education in values through physical-sports activities in young schoolchildren, and the evaluation of selfcontrol through the child and adolescent self-control questionnaire (CACIA). The results will help to evaluate whether the Delphi intervention program is appropriate for the development of values in students wherever the research is applied. This research aims to provide the teacher with tools for the real development of their students in terms of values through physical-sports activities. Likewise, the present work seeks to create a methodology that allows to transfer the educational values obtained in physical education classes to the family and society.
\end{abstract}

Key Words: Delphi program, physical education, aggressiveness, behaviors, values.

\section{Introducción}

Desde hace muchos años, se asume que la práctica deportiva desarrolla la moralidad y el carácter. Esta visión de la actividad deportiva se encuentra en los orígenes del ideario Olímpico y ha sido el motor impulsor del deporte moderno tal y como lo conocemos hoy. En cambio, un grupo de autores desmienten que la práctica deportiva por sí sola desarrolla el carácter, el trabajo en equipo, la cooperación y el respeto (Fejgin, 1994; Gruber, 1984; Shields y Bredemeier, 1995; Willis \& Campbell, 1992). Esto no significa que tales potenciales que se vivencian en la práctica deportiva no existan, sino que es arriesgado asumir que esos resultados se obtengan automáticamente por la participación. La ad-

Fecha recepción: 20-02-21. Fecha de aceptación: 02-02-22

Eulisis Smith Palacio

e.smith@ufv.es quisición de valores y las actitudes ocurrirán con mayor viabilidad, si se planifican tareas didácticas y herramientas pedagógicas que respondan a este fin en el marco de las actividades deportivas y la educación física (Gutiérrez, 2014; Smith-Palacio \& Bermejo- García, 2021).

La teoría del aprendizaje social y la aproximación al aprendizaje estructural son las bases de la investigación empírica del desarrollo moral en el deporte (Cecchini, Montero \& Peña, 2003; Carreres-Ponsoda, Escartí, Jiménez-Olmedo, \& Cortell-Tormo, 2021; Mujica \& Orellana, 2021). El desarrollo moral es consecuencia del modelado y reforzamiento de adultos significativos, de tal manera que el niño es capaz de asumir un rol en dependencia de la influencia externas provocadas por los padres, entrenadores y educadores (Bandura, 1986; Burton \& Kunce, 1995). Las regulaciones sociales y las normas movilizan el comportamiento de los discentes, a partir de los agentes socializadores ya sea a través de la escolarización o mediante las actividades deportivas. 
Por otra parte, la teoría del desarrollo estructural reúne toda su atención en la razón de los individuos, en juicio de valores y los comportamientos. Por tanto, una estructura de razonamiento moral está reflejada por el juicio propio, sobre cuál es el comportamiento correcto en un acto determinado y cuál no. Como resultado de la maduración cognitiva y las interrelaciones sociales esta estructura de razonamiento cambia, adaptándose a las situaciones de su entorno (Gilligan, 1982; Haan, 1991; Kohlberg, 1976; Piaget, 1965).

Diferentes investigaciones atestiguan la existencia del deporte como una filosofía de vida, llegando incluso a afirmar que el deporte es el mayor movimiento educativo internacional, siempre que en su contenido se especifiquen tareas concretas dirigidas al desarrollo moral de los estudiantes (Barker, Barker-Ruchti, Rynne \& Lee, 2012; Mountakis, 2000; Peneva, 2009; Rey \& Rubio, 2012). Otros autores hacen énfasis en la transferencia de los valores que se aprenden en el marco deportivo a la familia o al ámbito docente (Binder, 2012; Gasparini, 2012; Sánchez \& Bada, 2012, Sánchez, Marcos, Alonso, González \& Chamorro, 2012). Para que todo esto pueda ser efectivo, se necesitan conocer cuáles son las metodologías pedagógicas facilitadoras de transferencias de valores educativos, que benefician este tipo de aprendizaje desde la educación física y el deporte (Cecchini, et al., 2008; Gómez, 2005; Ruiz \& Cabrera, 2004; Whitehead, Telfer \& Lambert, 2013). Metodologías innovadoras recientes como el aprendizaje basado en problemas (Larmer, Mergendoller \& Boss, 2015), pretenden dar solución desde el punto de vista moral y dialógico a la formación de los escolares. En consecuencia, se pone al alcance de la comunidad educativa, un salto de la educación física, con nueva manera de interpretar y de aplicar el conocimiento científico, en este sentido podemos citar aprendizajes activos como: estudios de casos, estudios compartidos, estudios dirigidos o método de experto (Robledo, Fidalgo, Arias \& Álvarez, 2015; Mora \& Velert, 2020).

El autocontrol, es la capacidad que poseen las personas para manejar situaciones difíciles, manteniendo un nivel emocional alto. Podríamos decir también, que el autocontrol es la habilidad para gestionar las respuestas que se originan dentro de los elementos cognoscitivos, experimentales, comportamentales, de expresión física y bioquímica en las personas (Brenner \& Salovey, 1997; Serrano \& García, 2010). En este mismo sentido podemos decir, que el autocontrol es un grupo de habilidades-emocionales con gran incidencia en el ambiente educativo y social a través de los procesos de aprendiza- jes alcanzados durante el periodo de educación familiar. Estos efectos de la crianza tienen una gran influencia en: 1. la inhibición de respuestas rápidas; 2. preferencia en el repertorio conductual y 3. puesta en marcha de conductas elegidas intencionalmente. Para Epstein (1997) el autocontrol es la repercusión de la relación entre dos comportamientos: controladores y controlados; acompañados por frecuentes esfuerzos que tienen que ver con el retardo del castigo y que supone actuaciones buscando la gratificación inmediata. Con mucha frecuencia, en el modelaje de la conducta de adolescentes se incluyen técnicas de mejora de los procesos de autocontrol, entendiendo que el desarrollo del autocontrol se realiza mediante técnicas o terapias cuyo objetivo es enseñar a las personas estrategias para regular y modificar su propia conducta (López, López \& Freixinos, 2003).

Según Baños \& Arrayales (2020), las clases de educación física pueden ser un espacio donde se pueda experimentar el desarrollo de buenas relaciones entre iguales, potenciar ambientes de igualdad-escucha, debates y recrear una experiencia de empatía. Todas estas cuestiones harán que los alumnos dispongan de una actitud positiva ante las actividades académica y ante el colegio en general. Estas experiencias son desarrollables a través de las clases de educación física, facilitando un clima de aprendizajes que refuercen el compromiso, el esfuerzo y la perseverancia, elementos sin duda determinante en el rendimiento académico. Por otro lado (Muñoz, Lavega, Costes, Damián \& Serna, 2020; Ibañez, Camacho \& Marín, 2021) indican que el desarrollo de los estados de ánimos y la autorregulación de las actitudes influyen de forma directa en los aspectos cognoscitivos y relacionales.

El presente trabajo se justifica por la necesidad de explicar de forma objetiva, una metodología que permita el desarrollo de programas de intervención educativa, que promuevan valores y desarrollen actitudes, utilizando la educación física y el deporte como medio.

\section{Objetivo}

Plantear un protocolo metodológico en la implementación del Programa Delfos, para medir los niveles de autocontrol: autocontrol criterial, autocontrol procesual, retroalimentación personal, retraso en la recompensa y sinceridad, a través de las clases de educación física en adolescentes. De esta manera, proporcionar a la comunidad científica un método para la adquisición de valores a través de la educación física. 


\section{Metodología/Diseño}

La presente investigación es un estudio de tipo experimental longitudinal pretest-posttest. Los participantes pertenecientes al objeto de la muestra estratificada, colaborarán en el estudio de manera voluntaria, participando en las clases de educación física con el Programa Delfos. Se administrará el cuestionario CACIA antes y después de la intervención. El profesor que lleve a cabo la intervención habrá sido formado previamente con el objetivo de unificar criterios formativos.

\section{Participantes del estudio}

Todos los participantes del estudio serán alumnos y alumnas de colegios españoles de $5^{\circ}$ y $6^{\circ}$ de primaria. Siendo su participación voluntaria, se realizará un muestreo estratificado en base a las diferentes variables: género (hombre o mujer) y tipo de colegio (privado, concertado y público). Se precisa una muestra mínima de 150 alumnos para cada estudio, se recomienda hacer el cálculo con el número general de alumnos del colegio con la herramienta G* Power en base al 95\% de fiabilidad. También se podría utilizar el muestreo irrestricto aleatorio, en este tipo de muestreo el n es seleccionado de una muestra de tamaño $\mathrm{N}$ de tal manera que cada muestra de tamaño $\mathrm{n}$ tiene la misma probabilidad de ser seleccionada. El objetivo que persigue está metodología de muestreo es hacer una inferencia acerca de una población en relación con la muestra.

\section{Herramientas de medida}

\section{Cuestionario CACIA}

Este cuestionario de autocontrol infantil, para niños y adolescentes entre 11 y 19 años, fue desarrollado por Capafón-Bonet y Moreno (1986). Sus dimensiones se centran en la evaluación del autocontrol considerado desde el punto de vista conductual, cuya base es precisamente el esfuerzo de las personas por cambiar su actitud. También mide el esfuerzo de las personas por modificar sus reacciones. El cuestionario está compuesto por cinco dimensiones: retroalimentación personal (RP), autocontrol criterial (AC), retraso en la recompensa (RR), autocontrol procesual (AP) y sinceridad (S). El cuestionario se compone de 89 ítems con dos opciones de respuesta (sí o no). De las cinco dimensiones, tres miden aspectos positivos (RP, RR y AC), una mide aspectos negativos (AP) y la última mide nivel sinceridad (S), que indica espontaneidad, impulsividad, independencia de normas y exigencia sociales.
La RP se compone por 21 ítems. Una puntuación elevada indica la capacidad para conocerse a sí mismo/ a, darse cuenta de sus actos propios, el interés por los motivos y razones que te hacen actuar de una manera determinada; El RR se compone de 19 ítems. Recoge la regulación sobre respuestas impulsivas, permite dar prioridad a lo urgente y a lo importante, dejando a un lado lo que apetece. Por tanto, las puntuaciones altas indican un alto nivel de organización en el trabajo y en las acciones. De forma general una persona con retraso en la recompensa no se dejaría llevar por los actos de impulsos: El AC se compone de 10 ítems. Refleja la capacidad de las personas para soportar situaciones dolorosas o desagradables, también recoge aspectos de la responsabilidad personal. Sujetos con una puntuación elevada tendría una buena resistencia al estrés y situaciones amenazantes, mostrándose responsables, donde otras personas se asustarían; El AP se compone de 25 ítems. Conformado principalmente por autoevaluación, autogratificación y autocastigo. Una alta puntuación significaría que existe mucha preocupación por siempre actuar bien, un gran miedo al fracaso, o un gran sentimiento al rechazo. También esta escala indica el grado de autoestima que tiene la persona sobre sí misma, ansiedad por el logro de la meta, una supervaloración de los objetivos; La S se compone de 14 ítems. Una baja puntuación en esta escala supondría una alta dependencia a las normas sociales, y una alta puntuación refleja espontaneidad, impulsividad, independencia de las normas.

Este cuestionario ha sido utilizado en diferentes investigaciones (Cecchini et al., 2008; López et al., 2003; Torres et al., 2008). La fiabilidad interna fue determinada al calcular el coeficiente alfa de Cronbach, para la escala de retroalimentación personal $=.79$; el autocontrol procesual $=.76$; el retraso en la recompensa $=.71$; el autocontrol criterial $=.50$ y la sinceridad $=.63$. Siendo consistente con el estudio original y satisfactorio, teniendo en cuenta la heterogeneidad de los aspectos implicados en cada escala en adolescentes.

\section{Manejo de los datos. Adquisición, gestión y trans- ferencia de los datos}

Todos los datos de los participantes se recogerán a través del cuestionario CACIA por sus profesores. Los participantes del estudio se identificarán bajo una nomenclatura anónima, en ningún momento aportarán referencias de carácter identificativo. El cuestionario será autoadministrado, la duración de la aplicación será 40 minutos, se planificará una sesión para la 
implementación del cuestionario. Tanto el grupo experimental como el grupo de control realizarán los cuestionarios pretest y posttest. Al grupo experimental se le aplicará el tratamiento con el programa de intervención educativa Delfos. Los documentos recogidos se mantendrán bajo estricta confidencialidad con el único propósito de su utilización en la investigación en curso. A su vez, se mantendrán en lugar seguro al amparo de la legislación vigente para tal efecto. El desarrollo paso a paso de toda la estructura de la investigación se puede encontrar en la Figura 1. Anexo.

Los datos obtenidos de los cuestionarios se filtrarán, tabularán y codificarán para su posterior análisis en Statistical Package for the Social Science (SPSS v21).

\section{Análisis estadístico}

Se recomienda someter los datos a un análisis inicial con el propósito de verificar el cumplimiento de los supuestos paramétricos para lo que se utilizarán las pruebas de Kolmogorov-Smirnov y Shapiro-Wilks (supuesto de normalidad) y Levene (supuesto de homogeneidad de varianzas). Posteriormente si se confirma el cumplimiento de los supuestos, se realizarán pruebas de contraste de hipótesis, en concreto el test $t$ de Student y el ANOVA simple, para verificar las hipótesis principales. En caso contrario se utilizarán las pruebas no paramétricas U de Mann-Whitney y Kruskal-Wallis. Para mayor profundidad del estudio y confirmar la diferencia por curso, género y entorno geográfico, se realizará un ANOVA simple y la prueba de «Chi» cuadrado $(\div 2)$, dependiendo del cumplimiento o no de los supuestos paramétricos. Las variables que se analizarán son: retroalimentación personal; retraso en la recompensa; autocontrol procesual, autocontrol criterial y sinceridad. La hipótesis alterna $\mathrm{H}_{1}$ es: «El programa Delfos de 20 sesiones de educación física, tras su implementación mejora las variables de «Retroalimentación Personal», «Retraso en la Recompensa», «Autocontrol Criterial», «Autocontrol Procesual»y «Sinceridad» mediante las mediciones realizadas con el cuestionario CACIA, antes y después.

\section{Discusión}

El autocontrol se ha relacionado a la prevención de patrones de conductas desadaptadas (Kazdis \& BuelaCasal, 1994; Earls, 1994; López \& Taveras, 2021); a la mejora del rendimiento académico y al tratamiento de la agresividad (López, López \& Freixinos, 2003; Cecchini, Fernández \& Loza, 2011), existe una necesi- dad de profundizar en el desarrollo de mecanismos que ayuden cada vez más a los alumnos y alumnas al desarrollo del autocontrol como un medio de desarrollo personal y social. En los hallazgos encontrados por Gralinski \& Koop, 1993; Hoffman, 1994, explican que existe un bajo nivel de autocontrol asociado a las conductas antisociales. De esta manera se evidenciará, que la incapacidad para retrasar la recompensa está presente en los comportamientos agresivos, estas actitudes se trasladan cada vez más a los campos deportivos, a las aulas y la sociedad de forma general, herramientas pedagógicas como la que presentamos aquí permite corregir estos tipos de actitudes y dar una respuesta en poco tiempo a esta dificultad mediante la educación física.

Las actividades físico-deportivas son un espacio ideal para desarrollar el autocontrol, siempre que se diseñen estrategias didácticas que permitan el aprendizaje moral a través de la educación física y el deporte (Bredemeier \& Schields, 1986; Ramos, Parra, Santos \& Cangas, 2020). En esta misma línea, para un grupo de autores, el autocontrol es en esencia un mecanismo que permite eliminar las actitudes agresivas en el deporte, mejora el fair play y las actitudes prosociales en adolescentes (Cecchini, Fernández \& Arruza, 2008), estos autores encontraron, una disminución: de los comportamientos violentos, violación de las reglas y las protestas. Disminuyeron en esta misma investigación, las opiniones centradas en la búsqueda de la victoria a cualquier precio. Estos resultados son importantes, porque descentran la actividad deportiva de la competitividad y se centra en la búsqueda del logro de metas sociales. Aumentó además, la satisfacción de la diversión deportiva por encima de los resultados. En fin, nos encontramos ante un nuevo paradigma del deporte, que lleva a la comunidad científica a la formulación de un deporte más educativo, empático, prosocial, vinculado con el razonamiento y la formación humana.

La estrategia que se plantea, de vinculación de programas educativos-sociales como es el programa Delfos con la mejora del autocontrol mediante sesiones práctica dentro de las clases de educación física es eficaz, lo corroboran los resultados de Cecchini, Losa, González \& Arruza, (2008); Méndez Gimenez, Cecchini Estrada, \& García, (2018); Sánchez-Alcaraz, López-Jaime, Valero-Valenzuela \& Gómez-Mármol, (2017) ya que:1. incide de forma directa en el desarrollo moral de los alumnos y alumnas; 2. incide en el desarrollo del razonamiento; 3. permite utilizar el debate y el conflicto como un medio para el aprendizaje. Así mismo pode- 
mos decir que, esta metodología permite transferir los conocimientos y aprendizajes que se obtiene en las clases de educación física al ámbito de la familia, el ámbito escolar y la sociedad, mediante las hojas de observación que se entregaran a los alumnos al finalizar cada sesión de clase.

\section{Conclusión}

Se esperan conseguir resultados significativos mediante la intervención del Programa Delfos de 20 sesiones en educación física y las diferentes dimensiones del autocontrol (AP, AC, RR, RP y S), así como un tamaño del efecto entre moderado y grande. En este sentido, encontrar resultados positivos en esta investigación validarían la hipótesis de que la intervención de 20 sesiones de educación física a través del programa Delfos mejorarían competencias de autocontrol en el alumnado. Así mismo, se deben promover programas de educación física que persigan profundizar en la incidencia del autocontrol sobre la regulación del aprendizaje y su manifestación en las aulas, la familia y el entorno social. Ampliar a la vez, el abanico de edades y comparar el estado de autocontrol en las diferentes etapas de la adolescencia seria la proyección futura de estos programas. Validar estos tipos de programas, evidencia que el campo de la actividad física y el deporte es un espacio ideal para el aprendizaje mediante la experiencia y el aporte significativo a la ciencia.

\section{Referencias}

Baños, R. \& Arrayale, E. (2020). Predicción del aburrimiento en la educación física a partir del clima motivacional. Retos: Nueva Tendencia en Deportes, Educación física y Recreación. 38, 83-88.

https: / /doi.org/10.47197/retos.v38i38.74301

Barker, D., Barker-Ruchiti, N., Rynne, S., \& Lee, J. (2012). Olympism as education: Analysing the learning experiences of elite athletes. Educational Review, 64(3), 369-384. https://doi.org/10.1080/ 00131911.2012 .665846

Bandura, A. (1986). Social foundations of thought and action: A social cognitive theory. American Psychological Association. Englewood Cliffs, NJ: Prentice-Hall.

Binder, D. (2012). Olympic values education: Evolution of a pedagogy. Educational Review, 63(3), 275-302. Doi: 10.1080/00131911.2012.676539. https: / /doi.org/ 10.1080/00131911.2012.676539

Bredemeier, B. \& Schields, L. (1986). Moral Growth
Among Athletes and Nonathletes: A comparative Analysis. The Journal of Genetic Psychology, 147(1), 718. https://doi.org/10.1080/ 00221325.1986 .9914475

Brenner, E. M. \& Salovey, P. (1997). Emotional Regulation during Childhood: development, interpesonal and individual condideration. In P. Salovey D. J. Sludyter (Eds.): Emotional developmental and emotional intelligences. (168-195). NewYork: Basic Boocks.

Burton, R. V. \& Kunce, L. (1995). Behavioral models of moral development: A brief history and integration. En W. M Kurtines y J. L. Gewirtz (Eds.), Moral development:An introduction(pp.141-171). Boston: Allyn and Bacon.

Capafón- Bonet, A. \& Moreno, S. (1998). Cuestionario de Auto- Control Infantil y Adolescente. España:Tea Ediciones.

Cecchini, C., Cecchini, J.A., Losa, F., \& Mesa, C. (2011). Repercusiones del programa Delfos sobre los niveles de agresividad en las clases de educación física: actividades colaborativas versus competitivas. Magister: Revista Miscelánea de Investigación, (24), 1121.

Cecchini, J. A., Losa, J. F., González, C., \& Arruza, J. A. (2008). Repercusiones del Programa Delfos de educación en valores a través del deporte en jóvenes escolares. Effect of the Delfos Program of Education in Values Thrugh Sport in Studentes. Revista Educación, 346, 167-186.

Cecchini, J. A., Losa, J. F., González, C., \& Arruza, J. A. (2008). Los procesos de transferencia en el programa Delfos. Revista Española de Educación física, 8(382) 83-98.

Cecchini, J. A., Motero, J., \& Peña, J.V (2003). Repercusiones del programa de intervención para desarrollar la responsabilidad personal y social de Hellison sobre los comportamientos de fair play y auto control. Psicothema, 15(4), 631-637.

Cecchini Estrada, J. A, González-Mesa, C., MéndezGiménez, A., Fernández-Río, J., Contreras-Jordán, O., \& Romero-Granados, S. (2008). Metas sociales y de logro, persistencia-esfuerzo e intenciones de práctica deportiva en el alumnado de Educación Física. Psicothema, 20(2), 260-265.

Carreres-Ponsoda, F., Escartí, A., Jimenez-Olmedo, J. M., \& Cortell-Tormo, J. M. (2021). Effects of a Teaching Personal and Social Responsibility Model Intervention in Competitive Youth Sport. Frontiers in Psychology, 12, 408. https: / / doi.org/10.3389/ fpsyg. 2021.624018 
Durán, F., Seguí, P. V., \& López, M. C. (2016). Eficacia del programa de Pensamiento Prosocial en Entornos Educativos para el desarrollo de la competencia social de adolescentes en situación de riesgo de inadaptación social. Información Psicológica, (110), 3950. https://doi.org/10.14635/IPSIC.2015.110.6

Earls, F. (1994). Opposicional defiant and conduct disorders. Rutter., Taylor, E., Herson, L. (eds), Child and adolescent psychiatry: modern approches. Blackwell scientific publications: London.

Haan, N. (1991). Moral development and action from a social contructivist perpective. En W. M Kurtines y J. L. Gewirtz (Eds.), Handbook of moral behavior and development, Vol. 1: Theory (pp. 251-273). Hillsdale, NJ: Erlbaum.

Hoffman, M. (1994). Discipline and internalization. Developmental Psychology, 30, 26-28. https://doi.org/ 10.1037/0012-1649.30.1.26

Kazdin, A. \& Buela-Casal, G. (1994). Conducta antisocial: evaluación, tratamiento y prevención en la infancia y adolescencia. Edit. Piramdes: Madrid.

Fejgin, N. (1994). Participation in high school competitive sport: A subversión of school mision or contribution to academic goals? Sociology of Sport Journal, 11, 211-230. https://doi.org/10.1123/ ssj.11.3.211

Epstein, R. (1997). Skinner as self-manager. Journal Applied Behaviur Analisis, 30, 545-568. https:// doi.org/10.1901/jaba.1997.30-545

Foppiano, G. (2013). Actividad Física y Deporte Orientado al Esfuerzo de las Habilidades Sociales en Sectores de Alta Vulnerabilidad. (Doctoral dissertation. Universidad de Concepción. Facultad de Educación).

Gasparini, W. (2012). Penser L'intégration Et L'éducation Par Le Sport En France: Réflexions Sur Les Catégories Dánalyse Sociale Et Politique, Movement y Sport Sciences, 4, 39-44.

Gruber, J. J. (1984). Physical activity and self-esteem development in children: A meta-analysis. American Academy of Physical Educatión Papers, 19, 30-48.

Gilligan. C. (1982). In a different voice: Psychological theory and women's development. Cambridge, MA: Harvard University Press.

Gómez, (2005). La enseñanza y el aprendizaje de los valores en la educación deportiva. International Journal of Medicine and Sciences of Physical Activity and Sport, 5(18), 89-99.

Gutiérrez, M. (2014). Relaciones entre el clima motivational, la experiencia en educación física y la motivación intrínseca de los alumnos. Retos: Nueva
Tendencia en Deportes, Educación física y Recreación. 26, 9-14. https://doi.org/10.47197/retos.v0i26.34387 Gralinski, J. \& Koop, C. (1993). Everyday rules for behaviur: mother's requests to youngs children. Developmental Psychology, 29, 573-584. https:// doi.org/10.1037/0012-1649.29.3.573

Kohlberg, L. (1976). Moral stages and moralization: The cognitive-developmental approach. En T. Lickona (Ed). Moral development and behavior:Theory, research, and social issues (pp 31-53). New York: Holt, Rinehart y Winston.

Larmer, J. Mergendoller, J. \& Boss, S. (2015). Setting the Standard for Project Based Learning. USA: ASCD.

Lamoneda-Prieto, J., Huertas-Delgado, F., CórdobaCaro, L. G., \& García-Preciado, A.V. (2015). Desarrollo de los componentes sociales de la deportividad en futbolistas alevines. Cuadernos de Psicología del Deporte, 15(2), 113-124. https://doi.org/10.4321/ S1578-84232015000200013

López, C., López, J. R., \& Freixinos, M. A. (2003). Retardo de la gratificación y autocontrol en jóvenes antisociales: características asociadas al género. Psicopatología, Clínico, Legal y Forense, 3(3), 5-21.

López y Tavera, (2021). Uso del aprendizaje cooperativo en educación física y su relación con la responsabilidad individual en estudiantes del nivel secundario. Retos: Nueva Tendencia en Deportes, Educación física y Recreación. 43,1-9. https://doi.org/10.47197/ retos.v43i0.82607

Ibañes, M., Camacho, P. y Martín, A., (2021). El fútbol como recurso educativo en el deporte formativo de la provincia de Sevilla. Retos: Nueva Tendencia en Deportes, Educación fisica y Recreación. 43, 488-494. https:/ /doi.org/10.47197/retos.v43i0.89491

Mountakis, C. (2000). Values that should be developed through Olympic education programmes. Physical Education-Sport-Health, 8(9), 17-29.

Mora, J. L., \& Velert, C. P. (2020). Las relaciones sociales y su papel en la motivación hacia la práctica de actividad física en adolescentes: Un enfoque cualitativo. Retos: nuevas tendencias en educación física, deporte y recreación, (37), 41-47.

Muñoz- Arroyave, V., Lavega- Burgués, P., Costes, A., Damian, S., \& Serna, J. (2020). Los juegos motores como un recurso pedagógico para favorecer la afectividad desde la educación física. Retos: Nueva Tendencia en Deportes, Educación Física y Recreación. 38, 166172. https://doi.org/10.47197/retos.v38i38.76556 Mujica; F. N. \& Orellana, N. C. (2021). Educación en valores por medio del deporte. Una perspectiva fi- 
losófica basada en Søren Kierkegaard. Retos: Nueva Tendencia en Deportes, Educación Física y Recreación. 42, 887-892.

Méndez Giménez, A., Cecchini Estrada, J. A., \& García Romero, C. (2018). Metas de Logro 3x2, Inteligencia Emocional y Relaciones Sociales en el Contexto de la Educación Física. Revista Iberoamericana de Diagnóstico y Evaluación Psicológica, 4.

Peneva, B. I. (2009). Functions of Olympic education in contemporary school. Sport Science, 2, 31-34.

Piaget, J. (1965). The moral judgement of the child. New York: The Free Press.

Ramos, R. T., Parra, J. M. A., Santos, J. G., \& Cangas, A. J. (2020). Validación y adaptación de la escala de control psicológico del profesor hacia las clases de educación física y su efecto sobre las frustraciones de las necesidades psicológicas básicas. Retos: nuevas tendencias en educación física, deporte y recreación, (37), 167-173.

Rey, C. \& Rubio, K. (2012). The understanding of Olympic values by Brazilian Olympic atletes. Internactional Journal of Humanities Social Sciences and Education, 1(12), 37-43.

Robledo, P. Fidalgo, R., Arias, O. \& Álvarez, M. L. (2015). Percepción de los estudiantes sobre el desarrollo de las competencias a través de diferentes metodologías activas. Revista Investigación Educativa, 33(2), 369-383.

Robledo P., Fidalgo, R., Arias O., \& Álvarez M. L. (2015). Percepción de los estudiantes sobre el desarrollo de competencias a través de diferentes metodologías activas. Revista de Investigación Educativa,33(2), 369-383. doi: 10.6018/rie.33.2.201381

Ruiz, G. \& Cabrera, D. (2004). Los valores en el deporte. Revista de Educación, 335, 9-19.

Sánchez-Oliva, D., Marcos, M. L., Alonso, D. A., González- Ponce, I., \& Chamorro, J. M. L. (2012). Análisis diferencial de la percepción del desarrollo de valores en las clases de educación física. Retos: Nuevas Tendencia en Educación fisica, Deporte y Recreación, (22), 9-12. https://doi.org/10.47197/ retos.v0i22.34574

Sánchez-Alcaraz, B. J., López-Jaime, G., ValeroValenzuela, A., \& Gómez-Mármol, A. (2017). Los programas de educación en valores a través de la educación física y el deporte. Actividad Física y $D e-$ porte, Ciencia y Profesión, 28(25), 45-58.

Sánchez, A. \& Bada, J. (2012). Aprendiendo a resolver conflictos en(desde) el deporte. En De la Torre, F., Conde, P. Méndez- Rocasolano, M. y Blanco, P (Eds.), La solu- ción estructural de los conflictos (ADR): Estudio para la formación técnica negociadora(pp.211-234). España: Aranzadi.

Smith-Palacio, E. \& Bermejo-García, S. (2021). Efectividad del desarrollo del autocontrol a través de la educación física. Revista Iberoamericana de Ciencias de la Actividad Física y el Deporte, 10(2), 126-139.doi: 10.24310/riccafd.2021v10i2.11160

Serrano, M. E. \& García, D. (2010). Inteligencia emocional: autocontrol en adolescentes estudiantes del último año de secundaria. Multiciencia, 10(3), 273280.

Shields, D. L. L., \& Bredemeier, B. J. L. (1995). Character development and physical activity. Human Kinetic Publishers.

Willis, J. D. \& Cambell, L. F. (1992). Exercice Psychology. Champaing, IL: Human Kinetics.

Whitehead, J. Telfer, H. y Lambert, J. (2013). Values in youth sport and physical education. Routledge. https:/ /doi.org/10.4324/9780203114155

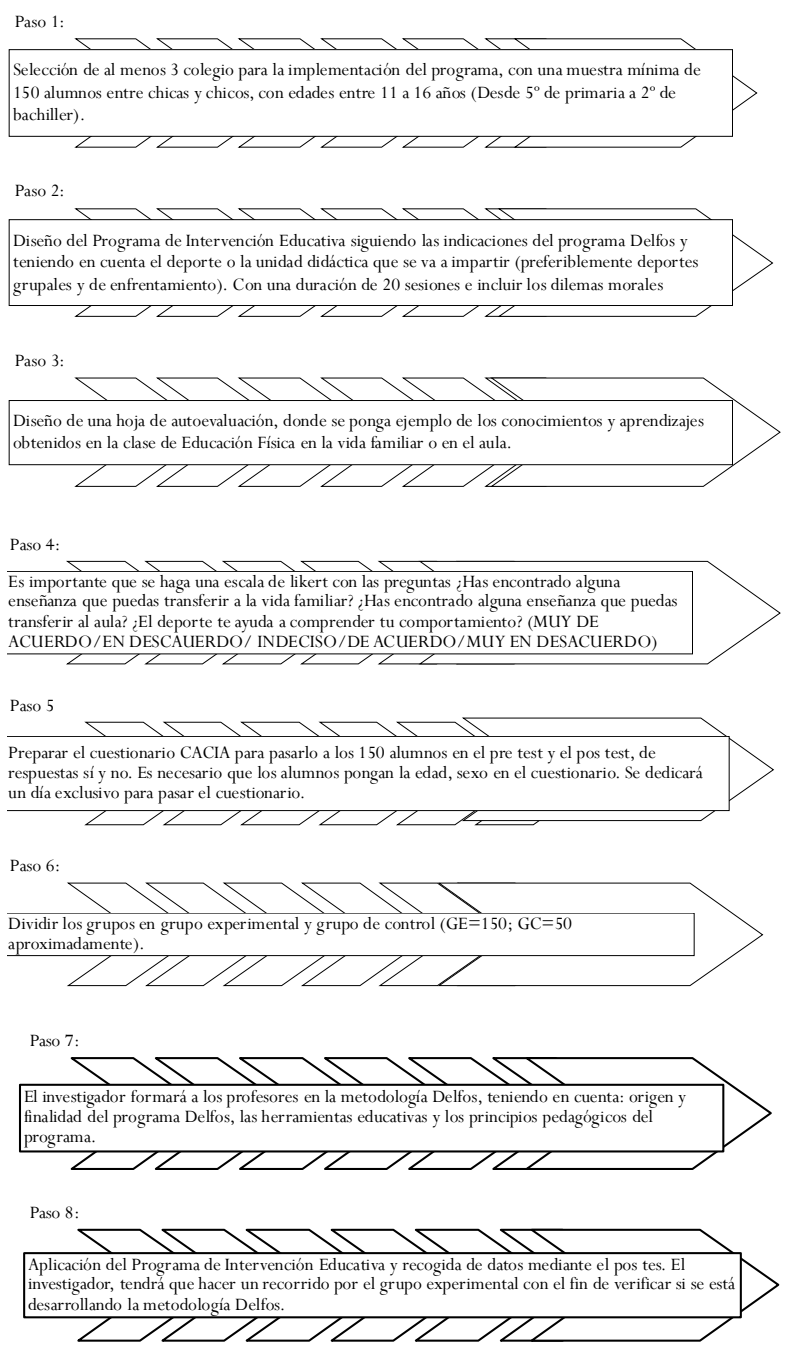

Figura 1. Descripción del proceso de la investigación 